\title{
Electron and Photon Reconstruction and Identification with the ATLAS Detector
}

\author{
Marine Kuna ${ }^{\mathrm{a}}$, on behalf of the ATLAS collaboration

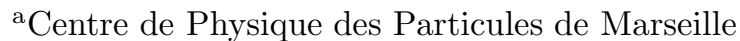 \\ 163, avenue de Luminy - Case 902 - 13288 Marseille cedex 09
}

This article presents the electron and photon reconstruction performance of the ATLAS detector based on the first LHC collision data at $\sqrt{s}=7 \mathrm{TeV}$. Calorimetric and tracker related electron identification variables are in a fair agreement with the Monte Carlo model describing the detector response. The position of the reconstructed photon conversions vertices has been used to compare the description of the inner detector used in Monte Carlo geometry to that from data. The energy flow measured in the electromagnetic calorimeter has been used to provide the same comparison at larger radii. $\pi^{0} \rightarrow \gamma \gamma$ and $J / \Psi \rightarrow e^{+} e^{-}$peaks were observed with reconstructed masses in good agreement with both Monte Carlo and PDG values. $17 \mathrm{~W} \rightarrow e \nu$ candidates and one $Z \rightarrow e^{+} e^{-}$ candidate have been observed in $6.69 \mathrm{nb}^{-1}$ of data.

\section{Electron and Photon Reconstruction in ATLAS}

The electron and photon reconstruction and identification in ATLAS $[1,2]$ rely mainly on the performance of the liquid argon-lead sampling electromagnetic calorimeter (EM calo) divided in a preshower and three longitudinal samplings, and the inner detector (ID) composed of 3 silicon pixel layers, 8 silicon strip layers providing 4 space points measurements and a transition radiation tracker (TRT) that, with an average of 30 hits per track, provides almost continuous tracking. The fraction of operational channels for each of these sub-detectors is above $97 \%$.

The identification of electrons consists of three series of rectangular cuts. The loose selection consists of cuts on the hadronic leakage and the shower shape in the $2^{\text {nd }}$ sampling. The medium set adds requirements on the shower shape in the $1^{\text {st }}$ sampling, track quality and cluster/track association. The tight cuts require further conditions on the fraction of high threshold TRT hits and adds a conversions veto. The identification cuts have been optimised with Monte Carlo simulations relying on a predicted detector geometry, its response to physics and a specific sample composition. The proportion of hadrons, electrons from conversions and prompt electrons (mostly from heavy quarks decay) of the electron candidates reconstructed from 10 millions events of non diffractive minimum bias Monte Carlo after each identification step are given in Table 1.

\begin{tabular}{c|c|c|c}
\hline & loose & medium & tight \\
\hline Hadrons & $68 \%$ & $66 \%$ & $30 \%$ \\
\hline$\gamma$-conversions & $28 \%$ & $23 \%$ & $22 \%$ \\
\hline Prompt electrons & $4 \%$ & $11 \%$ & $48 \%$ \\
\hline
\end{tabular}

Table 1

Composition of the electron candidates in hadrons, electrons from conversions and prompt electrons derived from non diffractive minimum bias Monte Carlo simulations after loose, medium and tight identifications.

128,909 preselected electrons from $\sim 1 \mathrm{nb}^{-1}$ of $\sqrt{s}=7 \mathrm{TeV}$ collisions data were compared to the simulation. They demonstrate a fair overall agreement for most of the variables [5]. On Figure 1 (top) is shown the data/Monte Carlo agreement for the fraction of high threshold TRT hits which is one of the most electron/hadron discriminating variables. The main disagreement for both electrons and photons [6] is observed (bottom) for showers in the $1^{\text {st }}$ and $2^{\text {nd }}$ calorimeter 

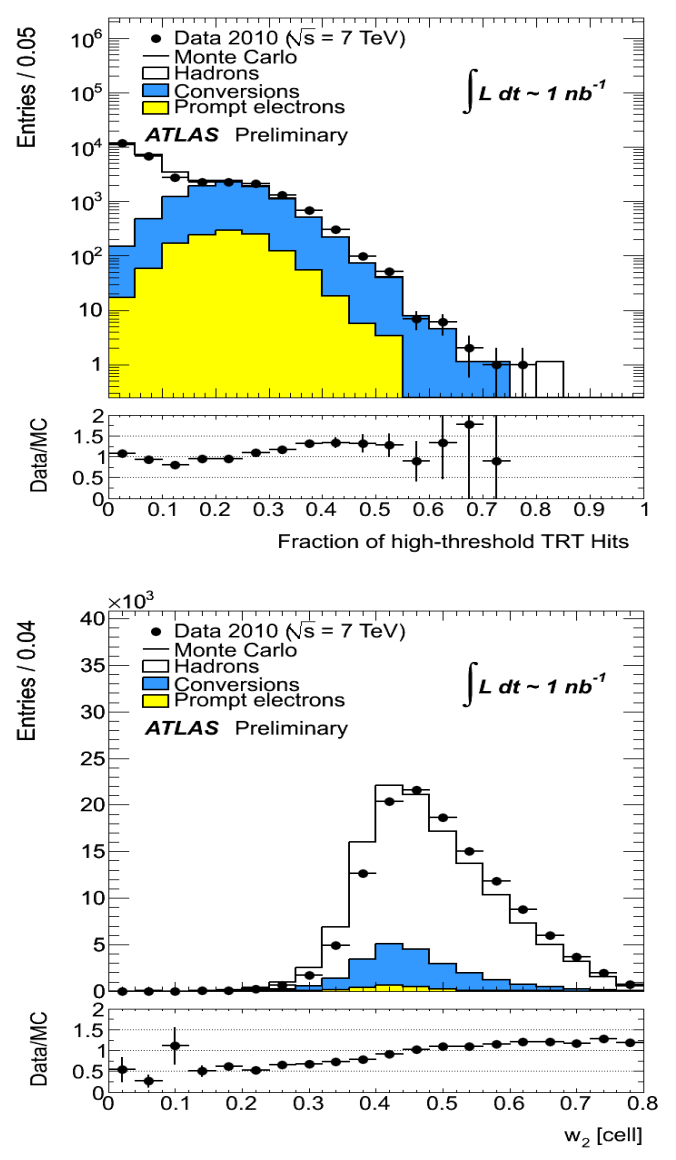

Figure 1. Top: Distribution of the fraction of high threshold TRT hits for electrons candidates passing the loose requirements. Bottom: Distribution of the energy weighted geometrical shower width in the $2^{\text {nd }}$ sampling of the EM calo for preselected electron candidates. the black dots represent $\sqrt{s}=7 \mathrm{TeV}$ collisions data. The histogram is the non diffractive minimum bias Monte Carlo divided in its different contributions : hadrons in white, electrons from photon conversions in blue and prompt electrons in yellow [4]. sampling that are wider in the data than in the Monte Carlo. Possible causes (simulation, cross talk, material), are currently under investigation.

\section{Material Mapping}

An optimal reconstruction of the electrons and photons in ATLAS requires the knowledge of the material upstream the electromagnetic calorimeter. The material estimation started with the first collisions data, using the reconstructed position of the photon conversion vertices to map the material in the ID and the energy flow in the EM calo to probe the total amount of material upstream of the EM calo.

In Figure 2 is shown the distribution of reconstructed photon conversion vertices as a function of the radius and longitudinal position for the $\sqrt{s}=7 \mathrm{TeV}$ collisions data. As expected, high conversion density is observed in regions corresponding to the pixel and SCT layers. Some data/simulation discrepancies could also be spotted like for instance the pixel global support ( $\sim 200 \mathrm{~mm}$ ) which was found shifted by $\sim 1 \mathrm{~cm}$ in radius.

The energy flow from minimum bias events [3] is an integral of the recorded energy in each cell of the EM calorimeter, summed over several events. It allows to check the EM calo response of all channels to physics signals. Its non-uniformity allowed to identify one readout cabling inversion and high voltage cable swaps that are now corrected for. The study also revealed up to $1 X_{0}$ underestimation of material in localised regions close to the rails supporting the ID. There was a good agreement for the rest of the barrel.

\section{3. $\pi^{0}, J / \Psi, \mathbf{W}$ and $\mathbf{Z}$ reconstruction}

The $\pi^{0} \rightarrow \gamma \gamma$ peak was extracted with clustering and calibration algorithms optimised for low energy $\gamma$. The reconstructed mass with $\sqrt{s}=7 \mathrm{TeV}$ collisions data $M_{\pi^{0}}=135.04+/-$ 0.04 agrees within $1 \%$ with both Monte Carlo and PDG values. The $\eta$ and $\phi$ uniformity show a data/MC agreement within $2 \%$ for barrel and endcaps [4]. The $\pi^{0}$ peak was also succesfully reconstructed with two converted photons, demon- 


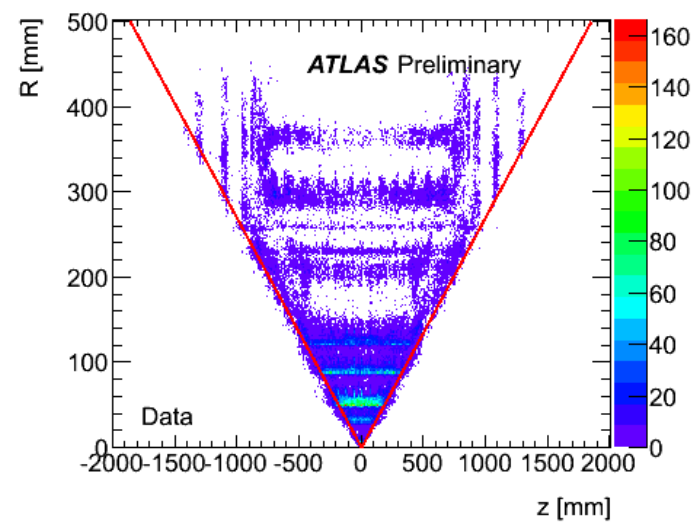

Figure 2. 2D distribution of reconstructed photon conversion vertices from $\sqrt{s}=7 \mathrm{TeV}$ collisions data as a function of the radius and longitudinal position [4].

strating the good performance of the ID.

The $J / \Psi \rightarrow e^{+} e^{-}$signal shown in Figure 3 was obtained with a cluster reconstruction optimised for low energies.

The standard requirements on cluster-track matching and discriminating variables (shower shape and track quality), as well as strict requirements on the fraction of high threshold hits in the Transition Radiation Tracker have been applied. The invariant mass has been computed using track parameters only. The tracks momenta have not been corrected for bremsstrahlung effects. The reconstructed mass of $M_{J / \Psi}=3052 \pm 65 \mathrm{MeV}$ and the width of $270 \pm 45 \mathrm{MeV}$ are in good agreement with the PDG value $M_{J / \Psi}=3096.916 \mathrm{MeV}$ [4].

A total integrated luminosity of $6.69 \mathrm{nb}^{-1}$ of $\sqrt{s}=7 \mathrm{TeV}$ collisions data with a full electron identification, a cut on the electron transverse energy $E_{T}^{\text {electrons }}>20 \mathrm{GeV}$, and a requirement on the missing transverse energy $E_{T}^{\text {miss }}>25 \mathrm{GeV}$ allowed the reconstruction of $17 \mathrm{~W} \rightarrow e \nu$ candidates for $21.9 \pm 0.6$ (stat) \pm 1.5 (syst) \pm 4.1 (lumi) expected events. One $Z \rightarrow e^{+} e^{-}$candidate has been observed with $1.6 \pm 0.1$ (syst) \pm 0.3 (lumi) expected.

\section{Conclusions}

The ATLAS electron and photon reconstruction and identification performance with early

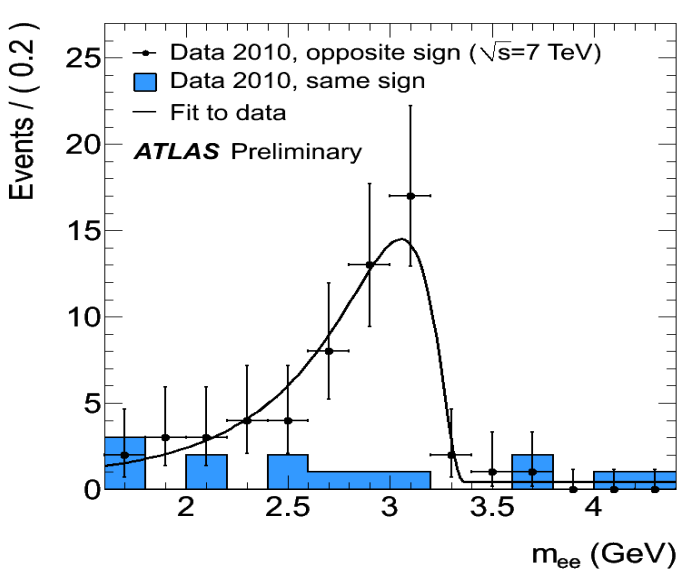

Figure 3. Reconstructed $J / \Psi \rightarrow e^{+} e^{-}$peak at $\sqrt{s}=7 \mathrm{TeV}[4]$.

$\sqrt{s}=7 \mathrm{TeV}$ collisions data have been presented. The very promising results represent a solid foundation towards the use of electrons and photons in the ATLAS physics program.

\section{REFERENCES}

1. ATLAS collaboration The ATLAS Experiment at the CERN Large Hadron Collider, 2008 JINST 3 S08003.

2. ATLAS collaboration Expected performance of the ATLAS experiment: detector, trigger and physics: Electrons and Photons, CERNOPEN-2008-020.

3. ATLAS Collaboration Probing the response of the ATLAS electromagnetic calorimeter and material upstream with energy flow from $\sqrt{s}=7 \mathrm{TeV}$ minimum bias events ATLASCONF-2010-037.

4. ATLAS Electron/Gamma public results webpage

https://twiki.cern.ch/twiki/bin/view/Atlas/

ElectronGammaPublicCollisionResults.

5. ATLAS collaboration Observation of prompt inclusive electrons in the ATLAS experiment at $\sqrt{s}=7$ TeV ATLAS-CONF-2010-073.

6. ATLAS collaboration Evidence for direct photon production in pp collisions at $\sqrt{s}=7 \mathrm{TeV}$ with the ATLAS detector ATLAS-CONF2010-077. 